\title{
Nonlinear symmetries of black hole entropy in gauged supergravity
}

\author{
Dietmar Klemm, ${ }^{a}$ Alessio Marrani, ${ }^{b, c}$ Nicolò Petri $^{a}$ and Marco Rabbiosi ${ }^{a}$ \\ ${ }^{a}$ Dipartimento di Fisica, Università di Milano, \\ and INFN, Sezione di Milano, \\ Via Celoria 16, I-20133 Milano, Italy \\ ${ }^{b}$ Museo Storico della Fisica e Centro Studi e Ricerche 'Enrico Fermi', \\ Via Panisperna 89A, I-00184 Roma, Italy \\ ${ }^{c}$ Dipartimento di Fisica e Astronomia 'Galileo Galilei', Università di Padova, \\ and INFN, Sezione di Padova, \\ Via Marzolo 8, I-35131 Padova, Italy \\ E-mail: dietmar.klemm@mi.infn.it, Alessio.Marrani@pd.infn.it, \\ nicolo.petri@mi.infn.it, marco.rabbiosi@mi.infn.it
}

ABSTRACT: Freudenthal duality in $N=2, D=4$ ungauged supergravity is generated by an anti-involutive operator that acts on the electromagnetic fluxes, and results to be a symmetry of the Bekenstein-Hawking entropy. We show that, with a suitable extension, this duality can be generalized to the abelian gauged case as well, even in presence of hypermultiplets. By defining Freudenthal duality along the scalar flow, one can prove that two configurations of charges and gaugings linked by the Freudenthal operator share the same set of values of the scalar fields at the black hole horizon. Consequently, Freudenthal duality is promoted to a nonlinear symmetry of the black hole entropy. We explicitly show this invariance for the model with prepotential $F=-i X^{0} X^{1}$ and Fayet-Iliopoulos gauging.

Keywords: Black Holes, Black Holes in String Theory, Supergravity Models

ARXIV EPRINT: 1701.08536 


\section{Contents}

1 Introduction 1

2 Attractors in gauged supergravity 3

3 Freudenthal duality $\quad 4$

3.1 Ungauged supergravity 4

3.2 U(1) FI-gauged $N=2, D=4$ supergravity 5

$\begin{array}{lll}3.3 & \text { Coupling to hypermultiplets } & 9\end{array}$

4 Final remarks $\quad 10$

\section{Introduction}

A consistent understanding of the microscopic origin of black hole entropy, and its relation to the macroscopic interpretation based on the Bekenstein-Hawking entropy-area formula, should be a key feature of any conceivable theory of quantum gravity. In this respect, string theory is successful, even if its accomplishments are currently limited mainly to extremal black holes that are asymptotically flat. In this framework, the black hole solutions, pertaining to the field theory limit in which supergravity arises, are described by configurations of wrapped D-brane states. The microscopic origin of the entropy then arises from state counting in a weakly coupled D-brane setup.

An important feature of supergravity black holes is the so-called attractor mechanism [1-5], describing the stabilization of the scalar fields near the event horizon only in terms of the conserved charges of the system, regardless of the initial conditions (asymptotic moduli) specified for their flow dynamics. This implies that the entropy does not depend on the asymptotic values of the scalar fields. After its discovery in asymptotically flat black holes in ungauged $N=2, D=4$ supergravity, the attractor mechanism has then been extended to the presence of abelian U(1) Fayet-Iliopoulos (FI) gauging [6-8] and to nontrivial hypermultiplets [9], where an abelian subgroup of the isometries of the quaternionic manifold was gauged.

Recent years have been characterized by an intense study of extremal black holes in gauged supergravity. This led e.g. to a symplectically covariant formulation of the equations satisfied by the solutions, of their attractor mechanism and scalar flow dynamics, as well as to the inclusion of coupling to hypermultiplets, cf. e.g. [9-19] for an (incomplete) list of references. In contrast with the case of ungauged theories in which hypermultiplets can always be consistently decoupled, in gauged supergravity hyperscalars may be charged and they actively participate to the solution. After [10], in which new solutions in gauged supergravities with nontrivial hypermultiplets were constructed by embedding 
known solutions of ungauged theories, further advances were made in [20], where the gauged supergravity analogue of the BPS attractor equations for theories coupled to hypermultiplets are derived and black holes with running hyperscalars are obtained numerically. In [9] the generalization of the effective black hole potential formalism [6] to abelian gaugings of the quaternionic hyperscalar manifold was given and in [21] a symplectically covariant formulation of the attractor mechanism and scalar flow dynamics in such a framework was achieved.

It is here worth remarking that hypermultiplets are essential in the formulation of realistic models given by the low energy limit of string/M-theory flux compactifications, which in turn are one of the most important motivations for the analysis of black hole solutions in gauged supergravity theories. Flux compactifications are indeed an effectively successful way to deal with the crucial moduli stabilization problem in string theory. The fluxes give rise to a nontrivial gauge potential in the effective theory, as well as to deformations determining gauged supergravity models in the low energy limit [22-24]. Thus, the study of the attractor mechanism within this scenario is of utmost importance $[25,26]$, because the presence of a charged black hole may drive the value of the moduli fields to an attractor horizon value which differs from the one obtained by the potential generated by flux compactification in the asymptotic region.

Recently, a novel symmetry was discovered for black holes in four-dimensional EinsteinMaxwell systems coupled to nonlinear sigma models of scalar fields (which can be regarded as the purely bosonic sector of an ungauged $D=4$ supergravity theory), namely the Freudenthal duality. It can be defined as an anti-involutive, nonlinear map acting on symplectic spaces, in particular on the representation space in which the electromagnetic charges of the black holes sit. After its introduction in [27] in the context of the so-called $U$-duality Lie groups of type $\mathrm{E}_{7}[28]$ in extended supergravity theories, interesting relations between Freudenthal duality, the Hessian matrix of the black hole entropy and the rigid special (pseudo-)Kähler metric of the prehomogeneous vector spaces associated to the $U$ orbits, were discovered and studied in [29, 30]. In [31] Freudenthal duality was proved to be a symmetry not only of the classical Bekenstein-Hawking entropy, but also of the critical points of the black hole potential. Moreover, it was consistently extended to any generalized special geometry, thus encompassing all $N>2$ (extended) supergravities, as well as $N=2$ generic special geometry, not necessarily having a coset space structure.

Interestingly, Freudenthal duality made its appearance also in a number of other contexts, such as gauge theories with symplectic scalar manifolds [32] and multi-centered black holes [33]. Moreover, Lagrangian densities exhibiting Freudenthal duality as an on-shell symmetry were constructed in [34] (in the context of black hole solutions in $N=2, D=4$ supergravity, see also [35]).

All the above formulations and results on Freudenthal duality were confined to ungauged theories. The present investigation is devoted to the consistent formulation of Freudenthal duality in the context of abelian gaugings of $N=2, D=4$ supergravity. This is done both for U(1) FI gauging and for theories coupled to hypermultiplets. As will be evident from the treatment given below, an essential ingredient for such generalizations is the effective black hole potential formalism introduced in $[6,9]$. 
In particular, Freudenthal duality will be proved to be an intrinsically nonlinear symmetry of the Bekenstein-Hawking extremal black hole entropy. Besides generalizing the correponding result in ungauged theories [27,31], this enlarges the set of invariance symmetries of the entropy function, thereby setting up the challenging question of the realization/interpretation of such an intrinsically non-linear symmetry in string/M-theory, also in the framework in which a Dirac-Zwanziger-Schwinger quantization condition for dyonic charges holds, and the symplectic representation space of electromagnetic fluxes is turned into a charge lattice [27].

The remainder of this paper is organized as follows: section 2 contains a brief review of the attractor mechanism in $N=2, D=4$ gauged supergravity coupled to vectorand hypermultiplets. Section 3 is devoted to the introduction of Freudenthal duality, starting with a summary of the ungauged case in 3.1. The extension to U(1) FI gauging is considered in section 3.2, and the further generalization to the coupling to hypermultiplets is presented in 3.3. We conclude in section 4 with some final remarks, hinting to further future developments.

Throughout this paper, we use the conventions of [21].

\section{$2 \quad$ Attractors in gauged supergravity}

In ungauged supergravity, the attractor mechanism [1-5] essentially states that, at the horizon of an extremal black hole, the scalar fields $\phi$ of the theory are always attracted to the same values $\phi_{\mathrm{h}}$ (fixed by the black hole charges $\mathcal{Q}$ ), independently of their values $\phi_{\infty}$ at infinity. When the so-called black hole potential has flat directions, it may happen that some moduli are not stabilized, i.e., their values at the horizon are not fixed in terms of the black hole charges. Yet, the Bekenstein-Hawking entropy turns out to be independent of these unstabilized moduli. Notice that this does not hold anymore for nonextremal black holes, for which the horizon is not necessarily an attractor point. The $\phi_{\mathrm{h}}$ are critical points of the black hole potential $V_{\mathrm{BH}}\left(\mathcal{Q}, z^{i}\right)$, where in $N=2, D=4$ supergravity the $z^{i}$ denote only the scalars in the vector multiplets, since hypermultiplets can be consistently decoupled. The horizon values $z_{\mathrm{h}}^{i}(\mathcal{Q})$ are thus determined by the criticality conditions

$$
\left.\partial_{i} V_{\mathrm{BH}}\left(\mathcal{Q}, z^{i}\right)\right|_{z_{\mathrm{h}}^{i}(\mathcal{Q})}=0
$$

and the Bekenstein-Hawking entropy is given by

$$
S_{\mathrm{BH}}=\left.\pi V_{\mathrm{BH}}\left(\mathcal{Q}, z^{i}\right)\right|_{z_{\mathrm{h}}^{i}(\mathcal{Q})} .
$$

In gauged supergravity, the scalar fields generically have a potential $V$, which contributes to the $\phi_{\mathrm{h}}(\mathcal{Q})$ as well. Both for U(1) Fayet-Iliopoulos gauging [6] and for abelian gauged hypermultiplets [9], the black hole potential in (2.1) has to be replaced by the effective potential

$$
V_{\mathrm{eff}}=\frac{\kappa-\sqrt{\kappa^{2}-4 V_{\mathrm{BH}} V}}{2 V},
$$

where $\kappa=0,1,-1$ corresponds to flat, spherical and hyperbolic horizons respectively. The limit for $V \rightarrow 0$ of $V_{\text {eff }}$ only exists for $\kappa=1$, in which case $V_{\text {eff }} \rightarrow V_{\mathrm{BH}}$, so one recovers 
correctly the black hole potential that governs the attractor mechanism in ungauged supergravity. The fact that this limit does not exist for $\kappa=0,-1$ is not surprising since flat or hyperbolic horizon geometries are incompatible with vanishing scalar potential. As before, the critical points of the effective potential determine the horizon values of the moduli,

$$
\left.\partial_{i} V_{\mathrm{eff}}\left(\mathcal{Q}, q^{u}, z^{i}\right)\right|_{z_{\mathrm{h}}^{i}, q_{\mathrm{h}}^{u}}=0,\left.\quad \partial_{u} V_{\mathrm{eff}}\left(\mathcal{Q}, q^{u}, z^{i}\right)\right|_{z_{\mathrm{h}}^{i}, q_{\mathrm{h}}^{u}}=0
$$

( $q^{u}$ are the hyperscalars), and the entropy density reads

$$
s_{\mathrm{BH}} \equiv \frac{S_{\mathrm{BH}}}{\operatorname{vol}(\Sigma)}=\frac{\left.V_{\mathrm{eff}}\left(\mathcal{Q}, q^{u}, z^{i}\right)\right|_{z_{\mathrm{h}}^{i}, q_{\mathrm{h}}^{u}}}{4}
$$

where $\Sigma$ denotes the unit $\mathrm{E}^{2}, \mathrm{~S}^{2}$ or $\mathrm{H}^{2}$.

\section{Freudenthal duality}

In this section we shall briefly review the Freudenthal duality in ungauged supergravity [27, $29,31]$, and subsequently generalize it to the gauged case.

\subsection{Ungauged supergravity}

Following [31], we introduce the scalar field dependent Freudenthal duality operator $\mathfrak{F}_{z}$ by

$$
\mathfrak{F}_{z}(\mathcal{Q}) \equiv \hat{\mathcal{Q}}=-\Omega \mathcal{M} \mathcal{Q}, \quad \mathfrak{F}_{z}(\mathcal{V}) \equiv \mathcal{V}
$$

where $\mathcal{Q}$ denotes the symplectic vector of charges, while the covariantly holomorphic symplectic section $\mathcal{V}$ and the matrices $\mathcal{M}, \Omega$ were defined in eqs. (2.1), (2.7) and (2.9) of [21] respectively. They satisfy the relations

$$
\mathcal{M}^{t}=\mathcal{M}, \quad \mathcal{M} \Omega \mathcal{M}=\Omega, \quad \mathcal{M V}=i \Omega \mathcal{V}, \quad \mathcal{M} D_{i} \mathcal{V}=-i \Omega D_{i} \mathcal{V}
$$

with $D_{i}$ the Kähler-covariant derivative. Moreover, the black hole potential can be written in terms of $\mathcal{Q}$ and $\mathcal{M}$ as

$$
V_{\mathrm{BH}}=-\frac{1}{2} \mathcal{Q}^{t} \mathcal{M} \mathcal{Q}
$$

As a consequence of (3.2), it follows that the action of $\mathfrak{F}_{z}$ on $\mathcal{Q}$ is anti-involutive, $\mathfrak{F}_{z}^{2}(\mathcal{Q})=$ $-\mathcal{Q}$. Using again (3.2), one shows that

$$
\mathfrak{F}_{z}\left(V_{\mathrm{BH}}\left(\mathcal{Q}, z^{i}\right)\right)=-\frac{1}{2} \hat{\mathcal{Q}}^{t} \mathcal{M} \hat{\mathcal{Q}}=V_{\mathrm{BH}}\left(\mathcal{Q}, z^{i}\right),
$$

i.e., the black hole potential is invariant under Freudenthal duality. Moreover, the second equation of (3.2) yields

$$
\partial_{i} \mathcal{M}=\mathcal{M} \Omega\left(\partial_{i} \mathcal{M}\right) \Omega \mathcal{M} .
$$

The direct application of this identity implies that under $\mathfrak{F}_{z}, \partial_{i} V_{\mathrm{BH}}$ flips sign, ${ }^{1}$

$$
\mathfrak{F}_{z}\left(\partial_{i} V_{\mathrm{BH}}\left(\mathcal{Q}, z^{i}\right)\right)=-\frac{1}{2} \hat{\mathcal{Q}}^{t}\left(\partial_{i} \mathcal{M}\right) \hat{\mathcal{Q}}=-\partial_{i} V_{\mathrm{BH}}\left(\mathcal{Q}, z^{i}\right) .
$$

\footnotetext{
${ }^{1}$ Since the operator $\mathfrak{F}_{z}$ does not commute with $\partial_{i}$, it is important to specify that $\mathfrak{F}_{z}$ acts always after the action of $\partial_{i}$. Notice that (3.6) corrects eq. (3.11) of [31].
} 
Since the $z_{\mathrm{h}}^{i}(\mathcal{Q})$ are the critical points of $V_{\mathrm{BH}}$, one has

$$
0=\left.\partial_{i} V_{\mathrm{BH}}\right|_{z_{\mathrm{h}}^{i}(\mathcal{Q})}=-\left.\mathfrak{F}_{z}\left(\partial_{i} V_{\mathrm{BH}}\right)\right|_{z_{\mathrm{h}}^{i}(\mathcal{Q})}=\left.\frac{1}{2} \hat{\mathcal{Q}}^{t}\left(\partial_{i} \mathcal{M}\right) \hat{\mathcal{Q}}\right|_{z_{\mathrm{h}}^{i}(\mathcal{Q})}=\frac{1}{2} \hat{\mathcal{Q}}_{\mathrm{h}}^{t} \partial_{i} \mathcal{M}\left(z_{\mathrm{h}}^{i}(\mathcal{Q})\right) \hat{\mathcal{Q}}_{\mathrm{h}}
$$

where we introduced Freudenthal duality $\mathfrak{F}$ at the horizon as

$$
\mathfrak{F}(\mathcal{Q})=\left.\mathfrak{F}_{z}(\mathcal{Q})\right|_{z_{\mathrm{h}}^{i}(\mathcal{Q})}=-\Omega \mathcal{M}_{\mathrm{h}} \mathcal{Q}=\hat{\mathcal{Q}}_{\mathrm{h}}
$$

On the other hand, applying (2.1) to the charge configuration $\hat{\mathcal{Q}}_{\mathrm{h}}$ leads to

$$
0=-\left.\partial_{i} V_{\mathrm{BH}}\left(\hat{\mathcal{Q}}_{\mathrm{h}}, z^{i}\right)\right|_{z_{\mathrm{h}}^{i}\left(\hat{\mathcal{Q}}_{\mathrm{h}}\right)}=\frac{1}{2} \hat{\mathcal{Q}}_{\mathrm{h}}^{t} \partial_{i} \mathcal{M}\left(z_{\mathrm{h}}^{i}\left(\hat{\mathcal{Q}}_{\mathrm{h}}\right)\right) \hat{\mathcal{Q}}_{\mathrm{h}} .
$$

Comparing (3.7) and (3.9), one can conclude that the attractor configuration

$$
z_{\mathrm{h}}^{i}\left(\hat{\mathcal{Q}}_{\mathrm{h}}\right)=z_{\mathrm{h}}^{i}(\mathcal{Q})
$$

is a solution also for (3.9) [31]. Eq. (3.10) can be interpreted as the stabilization of the near horizon configuration under Freudenthal duality, but an explicit verification of this claim is possible only if all the charges are different from zero. In any case one can always verify that $z_{\mathrm{h}}^{i}$ is critical point for both $V_{\mathrm{BH}}\left(\mathcal{Q}, z^{i}\right)$ and $V_{\mathrm{BH}}\left(\hat{\mathcal{Q}}_{\mathrm{h}}, z^{i}\right)$.

This fact turns out to be crucial in order to extend (3.1) to a symmetry of the black hole entropy $S_{\mathrm{BH}}$. In fact, using (2.2), (3.4) and (3.10), one obtains

$$
\begin{aligned}
\frac{1}{\pi} \mathfrak{F}\left(S_{\mathrm{BH}}\right) & =\mathfrak{F}\left(-\frac{1}{2} \mathcal{Q}^{t} \mathcal{M}\left(z_{\mathrm{h}}^{i}(\mathcal{Q})\right) \mathcal{Q}\right)=-\frac{1}{2} \hat{\mathcal{Q}}_{\mathrm{h}}^{t} \mathcal{M}\left(z_{\mathrm{h}}^{i}\left(\hat{\mathcal{Q}}_{\mathrm{h}}\right)\right) \hat{\mathcal{Q}}_{\mathrm{h}} \\
& =-\frac{1}{2} \mathcal{Q}^{t} \mathcal{M}_{\mathrm{h}} \mathcal{Q}=\frac{S_{\mathrm{BH}}}{\pi} .
\end{aligned}
$$

Thus, the entropy pertaining to the charge configuration $\mathcal{Q}$ is the same as the one pertaining to the Freudenthal dual configuration $\mathfrak{F}(\mathcal{Q})$. Since $\mathfrak{F}(\mathcal{Q})$ in $(3.8)$ is homogeneous of degree one (but generally nonlinear) in $\mathcal{Q},(3.11)$ results in the quite remarkable fact that the Bekenstein-Hawking entropy of a black hole in ungauged supergravity is invariant under an intrinsically nonlinear map acting on charge configurations. Note that no assumption has been made on the underlying special Kähler geometry, nor did we use supersymmetry.

\section{$3.2 \mathrm{U}(1)$ FI-gauged $N=2, D=4$ supergravity}

In U(1) FI-gauged $N=2, D=4$ supergravity, the parameters in terms of which the scalars $z^{i}$ stabilize at the horizon, are doubled by the gauge couplings $\mathcal{G}$. The entropy density and the horizon values of the scalars are now determined by the effective potential (2.3), which contains both $V_{\mathrm{BH}}$ and the scalar potential $V$.

As a first step, we extend the action of the field-dependent Freudenthal duality $\mathfrak{F}_{z}$ by acting on both $\mathcal{Q}$ and $\mathcal{G}$ according to

$$
\mathfrak{F}_{z}(\mathcal{Q})=\hat{\mathcal{Q}}=-\Omega \mathcal{M} \mathcal{Q}, \quad \mathfrak{F}_{z}(\mathcal{G})=\hat{\mathcal{G}}=-\Omega \mathcal{M G},
$$


while, by definition, $\mathfrak{F}_{z}$ leaves the symplectic section $\mathcal{V}$ (and its covariant derivatives) invariant. Now use (3.2), (3.5), and the fact that the scalar potential can be written as $[8,21]$

$$
V=g^{i \bar{\jmath}} D_{i} \mathcal{L} \bar{D}_{\bar{\jmath}} \overline{\mathcal{L}}-3|\mathcal{L}|^{2}=-\frac{1}{2} \mathcal{G}^{t} \mathcal{M G}-4|\mathcal{L}|^{2}
$$

where

$$
\mathcal{L} \equiv \mathcal{G}^{t} \Omega \mathcal{V}=\langle\mathcal{G}, \mathcal{V}\rangle
$$

to obtain

$$
\begin{aligned}
\mathfrak{F}_{z}\left(V\left(\mathcal{G}, z^{i}\right)\right) & =-\frac{1}{2} \hat{\mathcal{G}}^{t} \mathcal{M} \hat{\mathcal{G}}-4 \hat{\mathcal{L}} \hat{\overline{\mathcal{L}}}=V\left(\mathcal{G}, z^{i}\right), \\
\mathfrak{F}_{z}\left(\partial_{i} V\left(\mathcal{G}, z^{i}\right)\right) & =-\frac{1}{2} \hat{\mathcal{G}}^{t}\left(\partial_{i} \mathcal{M}\right) \hat{\mathcal{G}}-4\left(D_{i} \hat{\mathcal{L}}\right) \hat{\overline{\mathcal{L}}}=-\partial_{i} V\left(\mathcal{G}, z^{i}\right) .
\end{aligned}
$$

Since $V_{\text {eff }}$ and $\partial_{i} V_{\text {eff }}$ (cf. (2.26) of [6]) can be written as functions of $V_{\mathrm{BH}}, V, \partial_{i} V_{\mathrm{BH}}$ and $\partial_{i} V,(3.15)$, together with (3.4) and (3.6) implies

$$
\mathfrak{F}_{z}\left(V_{\mathrm{eff}}\left(\mathcal{Q}, \mathcal{G}, z^{i}\right)\right)=V_{\mathrm{eff}}\left(\mathcal{Q}, \mathcal{G}, z^{i}\right), \quad \mathfrak{F}_{z}\left(\partial_{i} V_{\mathrm{eff}}\left(\mathcal{Q}, \mathcal{G}, z^{i}\right)\right)=-\partial_{i} V_{\mathrm{eff}}\left(\mathcal{Q}, \mathcal{G}, z^{i}\right) .
$$

Using the second relation of (3.16), one has then

$$
\begin{aligned}
0 & =-\left.\partial_{i} V_{\text {eff }}\right|_{z_{\mathrm{h}}^{i}(\mathcal{Q}, \mathcal{G})}=\left.\mathfrak{F}_{z}\left(\partial_{i} V_{\text {eff }}\right)\right|_{z_{\mathrm{h}}^{i}(\mathcal{Q}, \mathcal{G})} \\
& =\left.\partial_{i} V_{\text {eff }}\left(\hat{\mathcal{Q}}, \hat{\mathcal{G}}, z^{i}\right)\right|_{z_{\mathrm{h}}^{i}(\mathcal{Q}, \mathcal{G})}=\partial_{i} V_{\text {eff }}\left(\hat{\mathcal{Q}}_{\mathrm{h}}, \hat{\mathcal{G}}_{\mathrm{h}}, z_{\mathrm{h}}^{i}(\mathcal{Q}, \mathcal{G})\right) .
\end{aligned}
$$

Let us define Freudenthal duality at the horizon by

$$
\begin{gathered}
\mathfrak{F}(\mathcal{Q})=\left.\mathfrak{F}_{z}(\mathcal{Q})\right|_{z_{\mathrm{h}}^{i}(\mathcal{Q}, \mathcal{G})}=-\Omega \mathcal{M}_{\mathrm{h}} \mathcal{Q}=\hat{\mathcal{Q}}_{\mathrm{h}}, \\
\mathfrak{F}(\mathcal{G})=\left.\mathfrak{F}_{z}(\mathcal{G})\right|_{z_{\mathrm{h}}^{i}(\mathcal{Q}, \mathcal{G})}=-\Omega \mathcal{M}_{\mathrm{h}} \mathcal{G}=\hat{\mathcal{G}}_{\mathrm{h}}
\end{gathered}
$$

From the comparison of (3.17) with the definition

$$
0=\left.\partial_{i} V_{\mathrm{eff}}\left(\hat{\mathcal{Q}}_{\mathrm{h}}, \hat{\mathcal{G}}_{\mathrm{h}}, z^{i}\right)\right|_{z_{\mathrm{h}}^{i}\left(\hat{\mathcal{Q}}_{\mathrm{h}}, \hat{\mathcal{G}}_{\mathrm{h}}\right)}=\partial_{i} V_{\mathrm{eff}}\left(\hat{\mathcal{Q}}_{\mathrm{h}}, \hat{\mathcal{G}}_{\mathrm{h}}, z_{\mathrm{h}}^{i}\left(\hat{\mathcal{Q}}_{\mathrm{h}}, \hat{\mathcal{G}}_{\mathrm{h}}\right)\right)
$$

it follows that

$$
z_{\mathrm{h}}^{i}\left(\hat{\mathcal{Q}}_{\mathrm{h}}, \hat{\mathcal{G}}_{\mathrm{h}}\right)=z_{\mathrm{h}}^{i}(\mathcal{Q}, \mathcal{G})
$$

is a solution also for (3.19), thus it is a critical point for both $V_{\text {eff }}$ and $\mathfrak{F}\left(V_{\text {eff }}\right)$.

Eqs. (2.5), (3.16) and (3.20) imply that $s_{\mathrm{BH}}$ is invariant under Freudenthal duality,

$$
\begin{aligned}
4 \mathfrak{F}\left(s_{\mathrm{BH}}\right) & =V_{\text {eff }}\left(\hat{\mathcal{Q}}_{\mathrm{h}}, \hat{\mathcal{G}}_{\mathrm{h}}, z_{\mathrm{h}}^{i}\left(\hat{\mathcal{Q}}_{\mathrm{h}}, \hat{\mathcal{G}}_{\mathrm{h}}\right)\right)=V_{\text {eff }}\left(\hat{\mathcal{Q}}_{\mathrm{h}}, \hat{\mathcal{G}}_{\mathrm{h}}, z_{\mathrm{h}}^{i}(\mathcal{Q}, \mathcal{G})\right) \\
& =V_{\text {eff }}\left(\mathcal{Q}, \mathcal{G}, z_{\mathrm{h}}^{i}(\mathcal{Q}, \mathcal{G})\right)=4 s_{\mathrm{BH}} .
\end{aligned}
$$

It is immediate to see that in the limit $\mathcal{G} \rightarrow 0$, one recovers the results of the ungauged case. Notice that the origin of Freudenthal duality is firmly rooted into the properties (3.2). The action of $\mathfrak{F}$ yields a new attractor-supporting configuration $\left(\hat{\mathcal{Q}}_{\mathrm{h}}, \hat{\mathcal{G}}_{\mathrm{h}}\right)$ that, in general, belongs to a physically different theory, specified by a different choice of gauge couplings.

It is worthwhile to note that no assumption has been made on the special Kähler geometry of the scalars in the vector multiplets. The invariance (3.21) holds thus also in 
models with non-homogeneous special Kähler manifolds, like e.g. the quantum stu model recently treated in [19].

As an illustrative example, let us check the action of Freudenthal duality for the simple model with prepotential $F=-i X^{0} X^{1}$ and purely electric FI gauging, cf. [7] for details. ${ }^{2}$ To keep things simple, we assume that the electric charges vanish. One has thus

$$
\mathcal{Q}=\left(\begin{array}{c}
p^{0} \\
p^{1} \\
0 \\
0
\end{array}\right), \quad \mathcal{G}=\left(\begin{array}{c}
0 \\
0 \\
g_{0} \\
g_{1}
\end{array}\right) .
$$

This model has just one complex scalar $z=x+i y$, and the matrix $\mathcal{M}$ is given by

$$
\mathcal{M}=\left(\begin{array}{cccc}
-\frac{x^{2}+y^{2}}{x} & 0 & \frac{y}{x} & 0 \\
0 & -\frac{1}{x} & 0 & -\frac{y}{x} \\
\frac{y}{x} & 0 & -\frac{1}{x} & 0 \\
0 & -\frac{y}{x} & 0 & -\frac{x^{2}+y^{2}}{x}
\end{array}\right) .
$$

The black hole and scalar potential read respectively

$$
\begin{aligned}
V_{\mathrm{BH}} & =-\frac{1}{2} \mathcal{Q}^{t} \mathcal{M} \mathcal{Q}=\frac{x^{2}+y^{2}}{2 x}\left(p^{0}\right)^{2}+\frac{\left(p^{1}\right)^{2}}{2 x}, \\
V & =-\frac{1}{2 x}\left(g_{0}^{2}+4 g_{0} g_{1} x+g_{1}^{2}\left(x^{2}+y^{2}\right)\right) .
\end{aligned}
$$

Plugging this into the effective potential (2.3), one shows that the latter is extremized for

$$
x=x_{\mathrm{h}}=\frac{u g_{0}}{g_{1}}, \quad y=y_{\mathrm{h}}=0,
$$

where $u$ is a solution of the quartic equation

$$
\left[\left(1-\nu^{2}\right) u+2\left(u^{2}-\nu^{2}\right)\right]^{2}=k\left(1-u^{2}\right)\left(\nu^{2}-u^{2}\right),
$$

with

$$
\nu \equiv \frac{g_{1} p^{1}}{g_{0} p^{0}}, \quad k \equiv \frac{\kappa^{2}}{\left(g_{0} p^{0}\right)^{2}} .
$$

Note that positivity of the kinetic terms in the action requires $x>0$. Depending on the sign of $g_{0} / g_{1}$, this means that either only negative or only positive roots of (3.26) are allowed, and such roots may not exist for all values of $\nu$ and $k$. Notice also that in the special case where

$$
\left(2 g_{0} p^{0}\right)^{2}=\left(2 g_{1} p^{1}\right)^{2}=\kappa^{2},
$$

the effective potential (2.3) becomes completely flat,

$$
V_{\mathrm{eff}}=-\frac{\kappa}{2 g_{0} g_{1}},
$$

\footnotetext{
${ }^{2}$ As discussed in section 10 of [36], the Freudenthal duality of $N=2, D=4$ supergravity minimally coupled to a certain number of vector multiplets in the ungauged case is nothing but a particular antiinvolutive symplectic transformation of the U-duality.
} 
and the scalar $z$ is thus not stabilized at the horizon, a fact first noted in [7]. (Nonetheless, the entropy is still independent of the arbitrary value $z_{\mathrm{h}}$, in agreement with the attractor mechanism). (3.28) corresponds to the BPS conditions found in [7], or to a sign-flipped modification of them. ${ }^{3}$ It would be interesting to see whether the appearance of flat directions is a generic feature of the BPS case, or just a consequence of the simplicity of the model under consideration. A large class of supersymmetric black holes in gauged supergravity satisfies a Dirac-type quantization condition [7] (that corresponds to a twisting of the dual superconformal field theory [37]), i.e., one has a relation between $\mathcal{Q}$ and $\mathcal{G}$, that enter into $V_{\mathrm{BH}}$ and $V$ respectively. This indicates that flat directions of $V_{\text {eff }}$ might be generic in the supersymmetric case.

Using (3.26), one can derive the near-horizon value of $V_{\text {eff }}$, and thus the entropy density (2.5),

$$
s_{\mathrm{BH}}=\frac{\left.V_{\mathrm{eff}}\left(\mathcal{Q}, \mathcal{G}, z^{i}\right)\right|_{z_{\mathrm{h}}^{i}(\mathcal{Q}, \mathcal{G})}}{4}=\frac{g_{0} p^{0^{2}}\left[\left(1-\nu^{2}\right) u+2\left(u^{2}-\nu^{2}\right)\right]}{4 \kappa g_{1}\left(1-u^{2}\right)} .
$$

We now determine the action of Freudenthal duality on the charges and the FI parameters. The definitions (3.18) yield

$$
\mathfrak{F}(\mathcal{Q}) \equiv\left(\begin{array}{c}
0 \\
0 \\
\hat{q}_{0} \\
\hat{q}_{1}
\end{array}\right)=\left(\begin{array}{c}
0 \\
0 \\
p^{0} x_{\mathrm{h}} \\
p^{1} / x_{\mathrm{h}}
\end{array}\right), \quad \mathfrak{F}(\mathcal{G}) \equiv\left(\begin{array}{c}
\hat{g}^{0} \\
\hat{g}^{1} \\
0 \\
0
\end{array}\right)=\left(\begin{array}{c}
-g_{0} / x_{\mathrm{h}} \\
-g_{1} x_{\mathrm{h}} \\
0 \\
0
\end{array}\right) .
$$

The dual configuration is thus electrically charged and has purely magnetic gaugings. For the transformed potentials one gets

$$
\begin{aligned}
\mathfrak{F}\left(V_{\mathrm{BH}}\right) & =-\frac{1}{2} \hat{\mathcal{Q}}_{\mathrm{h}}^{t} \mathcal{M} \hat{\mathcal{Q}}_{\mathrm{h}}=\frac{x^{2}+y^{2}}{2 x} \hat{q}_{1}^{2}+\frac{\hat{q}_{0}^{2}}{2 x}, \\
\mathfrak{F}(V) & =-\frac{1}{2} \hat{\mathcal{G}}_{\mathrm{h}}^{t} \mathcal{M} \hat{\mathcal{G}}_{\mathrm{h}}-4\left|\left\langle\hat{\mathcal{G}}_{\mathrm{h}}, \mathcal{V}\right\rangle\right|^{2}=-\frac{1}{2 x}\left(\left(\hat{g}^{1}\right)^{2}+4 \hat{g}^{0} \hat{g}^{1} x+\left(\hat{g}^{0}\right)^{2}\left(x^{2}+y^{2}\right)\right) .
\end{aligned}
$$

These are identical to (3.24), except for the replacements

$$
\left(p^{0}\right)^{2} \rightarrow \hat{q}_{1}^{2}, \quad\left(p^{1}\right)^{2} \rightarrow \hat{q}_{0}^{2}, \quad g_{0}^{2} \rightarrow\left(\hat{g}^{1}\right)^{2}, \quad g_{1}^{2} \rightarrow\left(\hat{g}^{0}\right)^{2}, \quad g_{0} g_{1} \rightarrow \hat{g}^{0} \hat{g}^{1} .
$$

The critical points of $\mathfrak{F}\left(V_{\text {eff }}\right)$ are thus $\hat{x}_{\mathrm{h}}=\hat{g}^{1} \hat{u} / \hat{g}^{0}$ and $\hat{y}_{\mathrm{h}}=0$, where $\hat{u}$ satisfies

$$
\left[\left(1-\hat{\nu}^{2}\right) \hat{u}+2\left(\hat{u}^{2}-\hat{\nu}^{2}\right)\right]^{2}=\hat{k}\left(1-\hat{u}^{2}\right)\left(\hat{\nu}^{2}-\hat{u}^{2}\right),
$$

with

$$
\hat{\nu} \equiv \frac{\hat{g}^{0} \hat{q}_{0}}{\hat{g}^{1} \hat{q}_{1}}, \quad \hat{k} \equiv \frac{\kappa^{2}}{\left(\hat{g}^{1} \hat{q}_{1}\right)^{2}} .
$$

Now, using (3.31), one easily shows that

$$
\hat{\nu}^{2}=\frac{1}{\nu^{2}}, \quad \hat{k}=\frac{k}{\nu^{2}} .
$$

\footnotetext{
${ }^{3}$ In the BPS case, $g_{0} p^{0}$ and $g_{1} p^{1}$ must have the same sign.
} 
Plugging this into (3.33) and multiplying with $\nu^{4} / \hat{u}^{4}$ yields

$$
\left[\left(1-\nu^{2}\right) \hat{u}^{-1}+2\left(\hat{u}^{-2}-\nu^{2}\right)\right]^{2}=k\left(1-\hat{u}^{-2}\right)\left(\nu^{2}-\hat{u}^{-2}\right) .
$$

Comparing with (3.26), we see that $u$ and $\hat{u}^{-1}$ satisfy the same equation, and have thus the same set of solutions. Hence, up to permutations of possible multiple roots, one gets $u=\hat{u}^{-1}$, which, by means of $(3.31)$, leads to $\hat{x}_{\mathrm{h}}=x_{\mathrm{h}}$, and therefore $V_{\text {eff }}$ and $\mathfrak{F}\left(V_{\text {eff }}\right)$ share the same critical points.

The transformed entropy density is given by

$$
\mathfrak{F}\left(s_{\mathrm{BH}}\right)=\frac{\left.V_{\mathrm{eff}}\left(\mathfrak{F}(\mathcal{Q}), \mathfrak{F}(\mathcal{G}), z^{i}\right)\right|_{\hat{z}_{\mathrm{h}}^{i}(\mathfrak{F}(\mathcal{Q}), \mathfrak{F}(\mathcal{G}))}}{4}=\frac{\hat{g}^{1} \hat{q}_{1}^{2}\left[\left(1-\hat{\nu}^{2}\right) \hat{u}+2\left(\hat{u}^{2}-\hat{\nu}^{2}\right)\right]}{4 \kappa \hat{g}^{0}\left(1-\hat{u}^{2}\right)} .
$$

Using again (3.31), it is easy to see that this coincides with (3.30), so that the entropy is indeed invariant under Freudenthal duality.

\subsection{Coupling to hypermultiplets}

In this section we generalize our analysis to include also hypermultiplets, and consider the case where abelian isometries of the quaternionic hyperscalar target manifold are gauged. The dynamics of the attractor mechanism is now governed by the potentials $V_{\mathrm{BH}}\left(\mathcal{Q}, z^{i}\right)$ and $V\left(\mathcal{P}^{x}\left(q^{u}\right), \mathcal{K}^{u}, z^{i}\right)$, where $\mathcal{P}^{x}=\left(\mathcal{P}^{x \Lambda}, \mathcal{P}_{\Lambda}^{x}\right)$ denote the triholomorphic moment maps, and $\mathcal{K}^{u}=\left(k^{\Lambda u}, k_{\Lambda}^{u}\right)$ are the Killing vectors that define the gauging. Note the presence of magnetic moment maps $\mathcal{P}^{x \Lambda}$ and magnetic Killing vectors $k^{\Lambda u}$. In what follows, we introduce the collective index $A=(i, u)$ and represent the scalars as

$$
\phi^{A}=\left(z^{i}, q^{u}\right) .
$$

As was shown in [21], the scalar potential can be written in the symplectically covariant form

$$
V=\mathbb{G}^{A B} \mathbb{D}_{A} \mathcal{L} \mathbb{D}_{B} \overline{\mathcal{L}}-3|\mathcal{L}|^{2}
$$

where

$$
\mathbb{G}^{A B}=\left(\begin{array}{cc}
g^{i \bar{\jmath}} & 0 \\
0 & h^{u v}
\end{array}\right), \quad \mathbb{D}_{A}=\left(\begin{array}{c}
D_{i} \\
\mathrm{D}_{u}
\end{array}\right), \quad \mathcal{L}=\left\langle\mathcal{Q}^{x} \mathcal{P}^{x}, \mathcal{V}\right\rangle, \quad \mathcal{Q}^{x}=\left\langle\mathcal{P}^{x}, \mathcal{Q}\right\rangle,
$$

provided the 'quantization condition' $\mathcal{Q}^{x} \mathcal{Q}^{x}=1$ holds. ${ }^{4}$

The field-dependent Freudenthal duality is again defined by (3.1), supplemented with

$$
\mathfrak{F}_{z}\left(\mathcal{P}^{x}\right) \equiv \hat{\mathcal{P}}^{x}=-\Omega \mathcal{M} \mathcal{P}^{x}, \quad \mathfrak{F}_{z}\left(\mathcal{K}^{u}\right) \equiv \hat{\mathcal{K}}^{u}=-\Omega \mathcal{M} \mathcal{K}^{u}
$$

One easily shows that $\mathfrak{F}_{z}\left(\mathcal{Q}^{x}\right)=\mathcal{Q}^{x}$ and, with slightly more effort, that

$$
\begin{aligned}
\mathfrak{F}_{z}\left(V_{\mathrm{eff}}\left(\mathcal{Q}, \mathcal{P}^{x}\left(q^{u}\right), \mathcal{K}^{u}\left(q^{u}\right), z^{i}\right)\right) & =V_{\mathrm{eff}}\left(\mathcal{Q}, \mathcal{P}^{x}\left(q^{u}\right), \mathcal{K}^{u}\left(q^{u}\right), z^{i}\right), \\
\mathfrak{F}_{z}\left(\partial_{A} V_{\mathrm{eff}}\left(\mathcal{Q}, \mathcal{P}^{x}\left(q^{u}\right), \mathcal{K}^{u}\left(q^{u}\right), z^{i}\right)\right) & =-\partial_{A} V_{\mathrm{eff}}\left(\mathcal{Q}, \mathcal{P}^{x}\left(q^{u}\right), \mathcal{K}^{u}\left(q^{u}\right), z^{i}\right) .
\end{aligned}
$$

\footnotetext{
${ }^{4}$ This represents a rather mild assumption, cf. footnote 8 of [21].
} 
Thus, in analogy to the U(1) FI case, one has to consider the criticality conditions (2.4) and apply the second relation of (3.40),

$$
\begin{aligned}
0 & =-\left.\left.\partial_{A} V_{\text {eff }}\left(\mathcal{Q}, \mathcal{P}^{x}, \mathcal{K}^{u}, z^{i}\right)\right|_{\phi_{\mathrm{h}}^{A}} \mathfrak{F}_{z}\left(\partial_{A} V_{\text {eff }}\left(\mathcal{Q}, \mathcal{P}^{x}, \mathcal{K}^{u}, z^{i}\right)\right)\right|_{\phi_{\mathrm{h}}^{A}}= \\
& =\left.\partial_{A} V_{\text {eff }}\left(\hat{\mathcal{Q}}, \hat{\mathcal{P}}^{x}, \hat{\mathcal{K}}^{u}, z^{i}\right)\right|_{\phi_{\mathrm{h}}^{A}}=\partial_{A} V_{\text {eff }}\left(\hat{\mathcal{Q}}_{\mathrm{h}}, \hat{\mathcal{P}}_{\mathrm{h}}^{x}\left(q_{\mathrm{h}}^{u}\right), \hat{\mathcal{K}}^{u}\left(q_{\mathrm{h}}^{u}\right), z_{\mathrm{h}}^{i}\right)
\end{aligned}
$$

where

$$
\hat{\mathcal{P}}_{\mathrm{h}}^{x}\left(q^{u}\right)=-\Omega \mathcal{M}_{\mathrm{h}} \mathcal{P}^{x}\left(q^{u}\right)
$$

is the dual expression for the moment maps that depends on the scalar fields, the charges and the parameters contained in the quaternionic Killing vectors. Defining $\hat{\mathcal{Q}}_{\mathrm{h}}$ as in (3.18), the criticality condition of the attractor points $\hat{\phi}_{\mathrm{h}}^{A}$ for the dual configuration of $\left(\mathcal{Q}, \mathcal{P}^{x}\left(q^{u}\right)\right)$, namely for $\left(\hat{\mathcal{Q}}_{\mathrm{h}}, \hat{\mathcal{P}}_{\mathrm{h}}^{x}\left(q^{u}\right)\right)$, reads

$$
0=\left.\partial_{A} V_{\mathrm{eff}}\left(\hat{\mathcal{Q}}_{\mathrm{h}}, \hat{\mathcal{P}}_{\mathrm{h}}^{x}, \hat{\mathcal{K}}^{u}, z^{i}\right)\right|_{\hat{\phi}_{\mathrm{h}}^{A}}=\partial_{A} V_{\mathrm{eff}}\left(\hat{\mathcal{Q}}_{\mathrm{h}}, \hat{\mathcal{P}}_{\mathrm{h}}^{x}\left(\hat{q}_{\mathrm{h}}^{u}\right), \hat{\mathcal{K}}^{u}\left(\hat{q}_{\mathrm{h}}^{u}\right), \hat{z}_{\mathrm{h}}^{i}\right) .
$$

Thus a comparison between (3.41) and (3.43) shows that the configuration

$$
\phi_{\mathrm{h}}^{A}=\hat{\phi}_{\mathrm{h}}^{A}
$$

is a solution for both criticality conditions. It follows that

$$
\begin{aligned}
4 \mathfrak{F}\left(s_{\mathrm{BH}}\right) & =V_{\text {eff }}\left(\hat{\mathcal{Q}}_{\mathrm{h}}, \hat{\mathcal{P}}_{\mathrm{h}}^{x}\left(\hat{q}_{\mathrm{h}}^{u}\right), \hat{z}_{\mathrm{h}}^{i}\right)=V_{\text {eff }}\left(\hat{\mathcal{Q}}_{\mathrm{h}}, \hat{\mathcal{P}}_{\mathrm{h}}^{x}\left(q_{\mathrm{h}}^{u}\right), z_{\mathrm{h}}^{i}\right) \\
& =V_{\text {eff }}\left(\mathcal{Q}, \mathcal{P}_{\mathrm{h}}^{x}\left(q_{\mathrm{h}}^{u}\right), z_{\mathrm{h}}^{i}\right)=4 s_{\mathrm{BH}},
\end{aligned}
$$

namely the entropy density of the two configurations related by the Freudenthal operator is the same.

\section{Final remarks}

In this paper, Freudenthal duality, a nonlinear anti-involutive map defined on the electromagnetic charge representation space of Einstein-Maxwell systems coupled to non-linear sigma models, was extended to the case where abelian isometries of $N=2, D=4$ supergravity coupled to vector- and hypermultiplets are gauged.

Without any assumption on the geometry of the scalar manifolds, the BekensteinHawking entropy was shown to be invariant under such a nonlinear map, which generally commutes with local supersymmetry (if any). As far as we know, this is the first example of a nonlinear symmetry of the black hole entropy itself, whose general invariance is usually given by the electromagnetic symplectic duality transformations, which act linearly on the charges and on the gauging parameters (within a symplectically covariant formalism).

Many further developments are possible, along the lines of the present investigation. We list and comment on some of them below.

As we pointed out, Freudenthal duality does not need supersymmetry, even if it was originally introduced in [27] in the context of $D=4$ supergravity theories with symmetric scalar manifolds. Along this venue of research, it would be interesting to extend the results presented above to abelian gaugings in theories with extended $(N>2)$ supersymmetry, 
and also to certain classes of $N=1$ models, whose symplectic structure is compatible with electromagnetic duality, thus allowing for an attractor behaviour of the near-horizon dynamics of the scalar flow (cfr. e.g. [38]).

Since its introduction, the stringy origin of Freudenthal duality has always remained a mistery, with its nonlinearity hinting to a nonperturbative nature. Also in view of the extension to the presence of gaugings - which generally characterize the supergravity theories obtained as low-energy limit of string and M-theory compactifications -, it would be interesting to deal with the challenging task of a realization of the Freudenthal antiinvolutive map in higher-dimensional string/M theory.

Finally, one can try to analyze the role and meaning of the intrinsically non-linear map provided by Freudenthal duality in string/M-theory flux compactifications, by using the AdS/CFT correspondence, especially in relation to recent results in which the large $N$ partition function of ABJM theory on spaces of the type $\Sigma \times \mathrm{S}^{1}$ was shown to reproduce the Bekenstein-Hawking entropy of static $\mathrm{AdS}_{4}$ BPS black holes [39]. Moreover, the role of the attractor mechanism for static black hole solutions in gauged supergravity coupled to hypermultiplets deserves further investigations. In particular, for both the models proposed in [40] and [41] to be dual to $\mathrm{AdS}_{4} \times V^{5,2} / \mathbb{Z}_{k}$, the field theory computations [42] show the same value of the topological free energy (up to a linear affine transformation of chemical potentials and charges). This fact may be related to Freudenthal duality and might point to some hidden link between the topological free energy and black hole entropy.

\section{Acknowledgments}

A. M. would like to thank the Physics Dept. of the University of Milan for kind hospitality and stimulating environment.

Open Access. This article is distributed under the terms of the Creative Commons Attribution License (CC-BY 4.0), which permits any use, distribution and reproduction in any medium, provided the original author(s) and source are credited.

\section{References}

[1] S. Ferrara, R. Kallosh and A. Strominger, $N=2$ extremal black holes, Phys. Rev. D 52 (1995) R5412 [hep-th/9508072] [INSPIRE].

[2] A. Strominger, Macroscopic entropy of $N=2$ extremal black holes, Phys. Lett. B 383 (1996) 39 [hep-th/9602111] [INSPIRE].

[3] S. Ferrara and R. Kallosh, Supersymmetry and attractors, Phys. Rev. D 54 (1996) 1514 [hep-th/9602136] [INSPIRE].

[4] S. Ferrara and R. Kallosh, Universality of supersymmetric attractors, Phys. Rev. D 54 (1996) 1525 [hep-th/9603090] [INSPIRE].

[5] S. Ferrara, G.W. Gibbons and R. Kallosh, Black holes and critical points in moduli space, Nucl. Phys. B 500 (1997) 75 [hep-th/9702103] [InSPIRE]. 
[6] S. Bellucci, S. Ferrara, A. Marrani and A. Yeranyan, $D=4$ Black Hole Attractors in $N=2$ Supergravity with Fayet-Iliopoulos Terms, Phys. Rev. D 77 (2008) 085027 [arXiv: 0802.0141] [INSPIRE].

[7] S.L. Cacciatori and D. Klemm, Supersymmetric AdS $S_{4}$ black holes and attractors, JHEP 01 (2010) 085 [arXiv:0911.4926] [INSPIRE].

[8] G. Dall'Agata and A. Gnecchi, Flow equations and attractors for black holes in $N=2 \mathrm{U}(1)$ gauged supergravity, JHEP 03 (2011) 037 [arXiv: 1012.3756] [INSPIRE].

[9] S. Chimento, D. Klemm and N. Petri, Supersymmetric black holes and attractors in gauged supergravity with hypermultiplets, JHEP 06 (2015) 150 [arXiv:1503.09055] [INSPIRE].

[10] K. Hristov, H. Looyestijn and S. Vandoren, BPS black holes in $N=2 D=4$ gauged supergravities, JHEP 08 (2010) 103 [arXiv: 1005.3650] [INSPIRE].

[11] K. Hristov and S. Vandoren, Static supersymmetric black holes in AdS 4 with spherical symmetry, JHEP 04 (2011) 047 [arXiv:1012.4314] [INSPIRE].

[12] D. Klemm and O. Vaughan, Nonextremal black holes in gauged supergravity and the real formulation of special geometry, JHEP 01 (2013) 053 [arXiv:1207.2679] [INSPIRE].

[13] C. Toldo and S. Vandoren, Static nonextremal AdS4 black hole solutions, JHEP 09 (2012) 048 [arXiv: 1207.3014] [INSPIRE].

[14] K. Hristov, S. Katmadas and V. Pozzoli, Ungauging black holes and hidden supercharges, JHEP 01 (2013) 110 [arXiv:1211.0035] [INSPIRE].

[15] D. Klemm and O. Vaughan, Nonextremal black holes in gauged supergravity and the real formulation of special geometry II, Class. Quant. Grav. 30 (2013) 065003 [arXiv:1211.1618] [INSPIRE].

[16] A. Gnecchi and C. Toldo, On the non-BPS first order flow in $N=2 \mathrm{U}(1)$-gauged Supergravity, JHEP 03 (2013) 088 [arXiv:1211.1966] [INSPIRE].

[17] D.D.K. Chow and G. Compère, Dyonic AdS black holes in maximal gauged supergravity, Phys. Rev. D 89 (2014) 065003 [arXiv:1311.1204] [InSPIRE].

[18] A. Gnecchi, K. Hristov, D. Klemm, C. Toldo and O. Vaughan, Rotating black holes in $4 d$ gauged supergravity, JHEP 01 (2014) 127 [arXiv:1311.1795] [INSPIRE].

[19] D. Klemm, A. Marrani, N. Petri and C. Santoli, BPS black holes in a non-homogeneous deformation of the STU model of $N=2, D=4$ gauged supergravity, JHEP 09 (2015) 205 [arXiv: 1507.05553] [INSPIRE].

[20] N. Halmagyi, M. Petrini and A. Zaffaroni, BPS black holes in AdS 4 from M-theory, JHEP 08 (2013) 124 [arXiv: 1305.0730] [INSPIRE].

[21] D. Klemm, N. Petri and M. Rabbiosi, Symplectically invariant flow equations for $N=2$, $D=4$ gauged supergravity with hypermultiplets, JHEP 04 (2016) 008 [arXiv:1602.01334] [INSPIRE].

[22] J. Polchinski and A. Strominger, New vacua for type-II string theory, Phys. Lett. B 388 (1996) 736 [hep-th/9510227] [INSPIRE].

[23] J. Michelson, Compactifications of type IIB strings to four-dimensions with nontrivial classical potential, Nucl. Phys. B 495 (1997) 127 [hep-th/9610151] [INSPIRE].

[24] G. Dall'Agata, Type IIB supergravity compactified on a Calabi-Yau manifold with H fluxes, JHEP 11 (2001) 005 [hep-th/0107264] [INSPIRE]. 
[25] J.P. Hsu, A. Maloney and A. Tomasiello, Black hole attractors and pure spinors, JHEP 09 (2006) 048 [hep-th/0602142] [INSPIRE].

[26] U.H. Danielsson, N. Johansson and M. Larfors, Stability of flux vacua in the presence of charged black holes, JHEP 09 (2006) 069 [hep-th/0605106] [INSPIRE].

[27] L. Borsten, D. Dahanayake, M.J. Duff and W. Rubens, Black holes admitting a Freudenthal dual, Phys. Rev. D 80 (2009) 026003 [arXiv:0903.5517] [InSPIRE].

[28] R.B. Brown, Groups of type $E_{7}$, J. Reine Angew. Math. 236 (1969) 79.

[29] S. Ferrara, A. Marrani, E. Orazi and M. Trigiante, Dualities Near the Horizon, JHEP 11 (2013) 056 [arXiv: 1305.2057] [INSPIRE].

[30] A. Marrani, Freudenthal Duality in Gravity: from Groups of Type $E_{7}$ to Pre-Homogeneous Spaces, arXiv: 1509.01031 [INSPIRE].

[31] S. Ferrara, A. Marrani and A. Yeranyan, Freudenthal Duality and Generalized Special Geometry, Phys. Lett. B 701 (2011) 640 [arXiv:1102.4857] [INSPIRE].

[32] A. Marrani, C.-X. Qiu, S.-Y.D. Shih, A. Tagliaferro and B. Zumino, Freudenthal Gauge Theory, JHEP 03 (2013) 132 [arXiv:1208.0013] [INSPIRE].

[33] J.J. Fernandez-Melgarejo and E. Torrente-Lujan, $N=2$ SUGRA BPS Multi-center solutions, quadratic prepotentials and Freudenthal transformations, JHEP 05 (2014) 081 [arXiv: 1310.4182] [INSPIRE].

[34] L. Borsten, M.J. Duff, S. Ferrara and A. Marrani, Freudenthal Dual Lagrangians, Class. Quant. Grav. 30 (2013) 235003 [arXiv:1212.3254] [INSPIRE].

[35] P. Galli, P. Meessen and T. Ortín, The Freudenthal gauge symmetry of the black holes of $N=2, d=4$ supergravity, JHEP 05 (2013) 011 [arXiv:1211.7296] [INSPIRE].

[36] S. Ferrara, R. Kallosh and A. Marrani, Degeneration of Groups of Type $E_{7}$ and Minimal Coupling in Supergravity, JHEP 06 (2012) 074 [arXiv:1202.1290] [INSPIRE].

[37] J.M. Maldacena and C. Núnez, Supergravity description of field theories on curved manifolds and a no go theorem, Int. J. Mod. Phys. A 16 (2001) 822 [hep-th/0007018] [INSPIRE].

[38] L. Andrianopoli, R. D'Auria, S. Ferrara and M. Trigiante, Black-hole attractors in $N=1$ supergravity, JHEP 07 (2007) 019 [hep-th/0703178] [INSPIRE].

[39] F. Benini, K. Hristov and A. Zaffaroni, Black hole microstates in $A d S_{4}$ from supersymmetric localization, JHEP 05 (2016) 054 [arXiv: 1511.04085] [INSPIRE].

[40] D. Martelli and J. Sparks, $A d S_{4} / C F T_{3}$ duals from M2-branes at hypersurface singularities and their deformations, JHEP 12 (2009) 017 [arXiv:0909.2036] [INSPIRE].

[41] D.L. Jafferis, Quantum corrections to $N=2$ Chern-Simons theories with flavor and their $A d S_{4}$ duals, JHEP 08 (2013) 046 [arXiv:0911.4324] [INSPIRE].

[42] S.M. Hosseini and N. Mekareeya, Large $N$ topologically twisted index: necklace quivers, dualities and Sasaki-Einstein spaces, JHEP 08 (2016) 089 [arXiv:1604.03397] [INSPIRE]. 